\title{
Temporale Bezüge bei Regulativen der Weiterbildungsbeteiligung
}

\author{
Empirische Befunde einer sozialräumlichen Studie
}

\section{Ewelina Mania}

Eingegangen: 30. Januar 2019 / Angenommen: 30. Mai 2019 / Online publiziert: 21. Juni 2019

(C) Der/die Autor(en) 2019

Zusammenfassung Zeit und Raum werden bislang in separaten Diskursen thematisiert - zumeist ohne dass dabei Verbindungen zwischen den Kategorien betrachtet werden. Eine integrierende Perspektive auf die Regulation der Weiterbildungsbeteiligung versprechen sozialraumorientierte Ansätze. Vor dem Hintergrund der Forderung der Verschränkung der Diskurse zu Zeit und Raum geht es in diesem Beitrag um die Frage, welche temporalen Bezüge sich in sozialräumlichen Regulativen der Weiterbildungsbeteiligung finden lassen. So werden zunächst die Kategorien Zeit und Raum skizziert, wobei es in erster Linie um ihre Bedeutung für die Forschung zu Weiterbildungsbeteiligung geht. Nach der Vorstellung des methodischen Hintergrunds werden anschließend im Sinne einer Verknüpfung von Zeit und Raum die Ergebnisse einer zeittheoretisch orientierten Analyse vorgestellt, bei der empirische Daten einer Studie zu sozialräumlichen Regulativen der (Nicht-)Teilnahme an organisierter Weiterbildung sogenannter ,bildungsferner Gruppen“ genutzt wurden.

Schlüsselwörter Weiterbildungsbeteiligung $\cdot$ Raum $\cdot$ Sozialraum $\cdot$ Zeit

\section{Temporal relationships in social space regulators of participation in further education \\ Empirical findings of a socio-spatial study}

Abstract So far, time and space have been addressed in separate discourses without considering the connections between the categories. An integrative perspective on the regulation of participation in further education promise social space perspective. Against the background of the demand for the interweaving of discourses of time 
and space, the paper deals with the question of which temporal relationships can be found in social space regulators of participation in further education. First of all, the categories of time and space are outlined, whereby their significance for research on participation in further education is primarily concerned. Following the presentation of the methodological background, the results of a time theory-oriented analysis are then presented in the context of a connection of time and space, in which empirical data of a study on social space regulators regarding participation of groups of less well-educated individuals were used.

Keywords Space $\cdot$ Social space $\cdot$ Time Adult education

\section{Einleitung}

Fragen der Weiterbildungsbeteiligung gehören spätestens seit der Wende zum 20. Jahrhundert zum Gegenstand der Adressaten-, Teilnehmer- und Zielgruppenforschung in der Erwachsenenbildung (vgl. Zeuner und Faulstich 2009). Unter den Begriffen „Weiterbildungsschere“ (vgl. Schulenberg et al. 1978), „Bildungsbenachteiligung“" (vgl. Brüning und Kuwan 2002) oder „Bildungsferne“ (vgl. Bremer et al. 2015) wird die Problematik der sozialen Selektivität der Weiterbildung diskutiert, wobei Zielgruppen thematisiert werden, die unterdurchschnittlich an Weiterbildung teilnehmen. Mit dem Begriff „Regulative der Weiterbildungsbeteiligung“ geht es im Anschluss an Wittpoth (2011) um hemmende oder begünstigende Einflüsse, welche für die Analyse des Zustandekommens der Weiterbildungsaktivitäten relevant sind. Diese Regulative werden in der Regel auf verschiedenen Ebenen untersucht, wobei soziodemografische Faktoren im Zentrum der Analysen stehen (vgl. ebd.).

Als ein Faktor, der immer wieder zur Erklärung der Weiterbildungsbeteiligung herangezogen wird, gilt die Kategorie Zeit. Lernen verläuft wie alle anderen Tätigkeiten in „temporalen Mustern“ (Faulstich 2001, S. 33) und ist in ,Temporalstrukturen verwickelt“ (Dörpinghaus 2008, S. 42). Zeit ist dabei ,weder subjektiv noch objektiv, sondern (...) als Schema beteiligt an der Sicht der Welt“ (Dörpinghaus 2008, S. 42). Die Vorstellung von Zeit als „Ressource“ gilt als selbstverständlich (vgl. Brödel und Yendell 2008, S. 56; Brödel et al. 1982). Der Faktor Zeit bzw. die Antwortmöglichkeit ,keine Zeit“" wird in diesem Zusammenhang als eine typische, sozial akzeptierte „Fluchtkategorie“" diskutiert, die selten zu validen Ergebnissen führt (vgl. Schmidt-Lauff 2018).

Begriffe wie „Lernzeiten“, „Zeitstrukturen“, „Zeitaufwand“, „Zeitverbrauch“ oder „Zeitvolumen“ verweisen auf die Vielfalt temporaler Bezüge. Schmidt-Lauff (2018, S. 335) bezeichnet Zeit insofern als eine Schlüsselkategorie in der Erwachsenenbildung und fordert eine ,zeitsensible“ Grundlegung der Erwachsenenbildung. Sie geht dabei von Zeit als einer umfassenden Kategorie aus, die vielfältige Aspekte und Dimensionen beinhaltet, die über ,bisherige Verwendungszusammenhänge von Zeit in der curricularen Verlaufsplanung, chronometrischen Lernsequenzgestaltung

\footnotetext{
1 Der Begriff „Fluchtkategorie“ bezieht sich nicht auf die gesellschaftliche Debatte zu Flucht und Migration.
} 
oder auch der Partizipationsforschung über Zeitstunden und Weiterbildungsteilnahme“ (ebd.) hinausgehen.

Zeit und Raum werden ,in der Regel singulär betrachtet, sodass ihre wechselseitige Verwobenheit und zwingenden Interdependenzen unsichtbar bleiben" (SchmidtLauff und Schreiber-Barsch 2018, S. 1). In den letzten Jahren werden integrierende und mehrdimensionale Modelle gefordert, die der Komplexität des Gegenstands gerecht werden (vgl. Bilger und Käpplinger 2017; Kaufmann und Widany 2013; Schemmann 2006; Wittpoth 2006; Holzer 2004). „Es geht darum, die bisher verfolgten Einzelperspektiven sowohl theoretisch als auch empirisch zusammenzubringen“" (Schemmann 2006, S. 31).

Eine integrierende Perspektive auf die Regulation der Weiterbildungsbeteiligung versprechen sozialraumorientierte Ansätze. Der Diskurs zum (Sozial-)Raum wird in den letzten Jahren in der Erziehungswissenschaft verstärkt geführt (vgl. Glaser et al. 2018). Auch in der Teildisziplin Erwachsenen- und Weiterbildung wird die Relevanz des Raumes für Theoriebildung und Praxis verstärkt diskutiert (vgl. Bernhard et al. 2015; Nuissl und Nuissl 2015; Stang et al. 2018).

Vor dem Hintergrund der Forderung nach einer Verschränkung der Diskurse zu Zeit und Raum geht es im Folgenden um die Frage, welche temporalen Bezüge sich in sozialräumlichen Regulativen der Weiterbildungsbeteiligung finden lassen? Damit soll ein Beitrag dazu geleistet werden, die beiden bisher isoliert geführten Stränge der Weiterbildungsbeteiligungsforschung zu verbinden. So werden zunächst die Kategorien Zeit und Raum skizziert, wobei es in erster Linie um ihre Bedeutung für die Forschung zu Weiterbildungsbeteiligung geht. Nach der Darlegung des methodischen Hintergrunds werden im Sinne einer Verknüpfung von Zeit und Raum die Ergebnisse einer zeittheoretisch orientierten Analyse vorgestellt, bei der empirische Daten einer Studie zu sozialräumlichen Regulativen der (Nicht-)Teilnahme an organisierter Weiterbildung ,bildungsferner Gruppen“ genutzt wurden.

\section{Zeit und Raum im Kontext von Regulativen der Weiterbildungsbeteiligung}

Die Thematisierung von Zeit und Raum erfolgt im Kontext der Regulative der Weiterbildungsbeteiligung bisher unverbunden. Im Folgenden wird der jeweilige Diskurs- bzw. Forschungsstand kurz skizziert.

\subsection{Zeit in der Forschung zur Weiterbildungsbeteiligung}

Im Rahmen der Untersuchungen zu Weiterbildungsbeteiligung wird Zeit vor allem als persönliche Ressource und pragmatische Größe gesehen. Betrachtet wird in erster Linie der zeitliche Umfang von Weiterbildungsangeboten, womit die Zeitspanne und die in Weiterbildungsaktivitäten investierte Zeit in Stunden gefasst wird (vgl. Bilger und Kuper 2013). Der Faktor Zeit wird zudem vor allem in repräsentativen bzw. standardisierten Erhebungen zu Weiterbildungsbeteiligung als Weiterbildungsbarriere und Grund für Nicht-Teilnahme an organisierter Weiterbildung gesehen. Die Antwortmöglichkeit „,keine Zeit/Zeitmangel“ wird dabei in der Regel am häufigsten 
gewählt (vgl. Bilger und Käpplinger 2017; Schiersmann 2006; Baethge und BaethgeKinsky 2004). Exemplarisch sei hier auf die Münchener-Studie (Tippelt et al. 2003, S. 58) verwiesen, bei welcher die Antwort ,ich hatte keine Zeit“ mit 42,4\% als häufigstes „Nichtteilnahmemotiv“ angegeben wurde. Im Rahmen des Adult Education Surveys (AES) wird mit Blick auf Zeitmangel zwischen ,beruflichen Terminen“ und „familiären Verpflichtungen“ unterschieden, die mit jeweils $42 \%$ und $39 \%$ auf Platz 1 und 2 der Gründe für eine Nicht-Teilnahme an organisierter Weiterbildung rangieren (Bilger und Käpplinger 2017, S. 270).

Vor dem Hintergrund des Konzepts des Lebenslangen Lernens stellt sich die Frage, inwiefern die Antwortmöglichkeit ,keine Zeit/Zeitmangel“ als eine „Fluchtkategorie“ (vgl. Schmidt-Lauff 2018; Barz und Tippelt 2004, S. 97) bzw. „Schutzbehauptung“ (Grotlüschen 2010, S. 85) zu sehen ist. Die Nutzung von Zeit als Fluchtkategorie hat zu Folge, dass ,,argumentative Ausführungen oder Begründungen kaum mehr nötig erscheinen (weder auf der subjektiven, noch auf der institutionellen oder politischen Ebene)“ (Schmidt-Lauff 2007, S. 223). Barz und Tippelt (2004, S. 97) vermuten hinter dem jeweiligen Zeitargument ,bestimmte Motivkonstellationen bzw. Prioritätensetzungen“, die sie mithilfe qualitativer Daten auf berufliche und familiäre Verpflichtungen sowie ,vielseitige Interessen“ und die Wertschätzung von „Freizeit und Hobbies“ beziehen. Daher liegt die Herausforderung für die Weiterbildungsbeteiligungsforschung darin, die Auswirkungen sozialer Erwünschtheit beim Antwortverhalten zu reduzieren (vgl. Kuwan 2011, S. 389). Bolder (2011, S. 60) verweist in dem Kontext auf die Diskrepanz zwischen durchgängiger Akzeptanz des Postulats „Lebenslangen Lernens“ auf der einen Seite und der (geringen) praktizierten Teilnahme an Weiterbildung auf der anderen Seite.

Während in vielen Arbeiten Zeit unter dem Blickwinkel von Zeitmangel betrachtet wird, gibt es auch Arbeiten, welche die Vielfalt temporaler Aspekte und Bezüge von Bildung und Lernen im Erwachsenenalter herausarbeiten (vgl. Schlüter 2005; Wolff 2005; Schmidt-Lauff 2012; Schäffter 1993). Ausgehend von einer Aufarbeitung unterschiedlicher zeittheoretischer Positionen und eigener empirischer Studien zeigt Schmidt-Lauff (2008, 2018) zeittheoretische Implikationen für die Erwachsenenbildung auf. In ihrem heuristischen Modell unterscheidet sie zum einen zwischen ,temporalen Grundbezügen“, mit welchen ,generelle und universelle Eigenheiten von Zeit" gemeint sind, wie Zeitverbrauch, Zeitverlauf, Emotionalität und Flüchtigkeit, um nur einige wenige Aspekte zu nennen (s. dazu ausführlich SchmidtLauff 2012). Zum anderen unterscheidet das Modell „Selbstverhältnisse zu Zeit“, die sich auf „subjektive Bewertungen aus den Erfahrungen dieser Grundbezüge“ beziehen (Schmidt-Lauff 2018, S. 325). Die „Selbstverhältnisse zu Zeit“ sind „in ihrer Widersprüchlichkeit und Komplexität zwar erfahrbar, aber schwer fassbar oder verbalisierbar" (ebd.). Die temporalen Grundzüge und die Selbstverhältnisse zu Zeit sind dabei nicht eins zu eins aufeinander bezogen, sondern in den ,Selbstverhältnissen zu Zeit" verbinden sich die temporalen Grundbezüge miteinander. SchmidtLauff (2018) benennt folgende „Selbstverhältnisse zu Zeit“:

1. Selbstverhältnis einer temporalen Dimensionsverschränkung (Lerninitiativen und Gründe zwischen Vergangenheit, Gegenwart und Zukunft) 
2. Selbstverhältnis expliziter Zeitanteile (Suche nach Zeitfenstern für Bildung und Lernen)

3. Selbstverhältnis der flexiblen Kontinuität (individuelle Flexibilität sowie Rahmungen zur Rhythmisierung von Lernen)

4. Selbstverhältnis temporaler Formalisierung (temporale Gegentendenzen zu (De-)Institutionalisierung und Selbststeuerung)

5. Selbstverhältnis struktureller und biografischer Zeitkonkurrenzen (Zeitverwendung zwischen Sozialzeit und Eigenzeit)

6. Selbstverhältnis einer Ökonomisierung von Lernzeit (zukünftige Verwertungsausrichtung und effizienzbetonte Prozessgestaltung)

7. Selbstverhältnis temporal divergenter Strukturen (Bildung und Lernen zwischen konfligierenden Zeitordnungen)

8. Selbstverhältnis von Zeit als Fluchtkategorie (Erleben der Vielschichtigkeit zeitlicher Strukturen und Erfahrungen, wie auch Nichtkommunizierbarkeit temporaler Eindrücke).

Diese Kategorisierung hebt die Vielfalt temporaler Elemente und Dimensionen hervor, die in der bisherigen Forschung zur Weiterbildungsbeteiligung kaum in den Blick genommen wurden. Diese Selbstverhältnisse werden in Kap. 4 als Gliederungsstruktur wieder aufgenommen und inhaltlich diskutiert.

\subsection{Raum in der Forschung zur Weiterbildungsbeteiligung}

Wie schon die Zeit stellt der Raum bzw. die Räumlichkeit ein keineswegs neues, „,sondern ein uraltes Thema pädagogischer Debatten und deren wissenschaftlicher Reflexion“ (Kessl 2016, S. 15) dar. So wurde die Kategorie Raum in der Erwachsenenbildung immer wieder thematisiert, ,bleibt aber insgesamt doch eher ein Randthema" (Stang et al. 2018, S. 644).

Insgesamt lassen sich drei Raumvorstellungen unterscheiden, die jeweils eine eigene Perspektive darstellen: absolutistische, relativistische und relationale Raumvorstellung (vgl. Fritsche et al. 2010). Während die absolutistische oder auch territoriale Raumvorstellung (vgl. Ebner von Eschenbach und Ludwig 2015) vom Raum als „Behälter“ ausgeht, in dem Körper enthalten sind, geht die relativistische Raumvorstellung vom Raum als „Ergebnis von Beziehungen zwischen Körpern“ aus (vgl. Fritsche et al. 2010, S. 13) und fokussiert die Handlungsebene. Sowohl die Vorstellung des absolutistischen Raums als auch der Gegenentwurf des relativistischen Raums erscheinen verkürzt (vgl. Kessl und Reutlinger 2009, S. 202). Die beiden Raumvorstellungen werden in einer relationalen Raumvorstellung aufgehoben, die sowohl den strukturellen Aspekt als auch den Handlungsaspekt berücksichtigt (vgl. Fritsche et al. 2010, S. 14). Im Anschluss an die Raumkonzeption von Löw (2001, S. 154) wird Raum als ,relationale (An)Ordnung von Lebewesen und sozialen Gütern" verstanden.

Hinsichtlich der bisherigen Raumbezüge in der Erwachsenenbildung zeigen sich verschiedene Verwendungsweisen (vgl. Hof 2014) und Rezeptionsstränge von Raum, sozialem Raum oder Sozialraum (vgl. Mania et al. 2015). Raumfragen werden beispielsweise auch unter dem Begriff „Region“ (vgl. Bernhard 2014), 
„Bildungslandschaft“ (vgl. Bleckmann und Schmidt 2011), „Lernort“ (vgl. Tippelt und Reich-Claassen 2010) oder „Lernraum“ (vgl. Stang 2015) diskutiert.

Mit Blick auf Raum als Regulativ der Weiterbildungsbeteiligung wurden bisher vor allem die materiell-infrastrukturellen Aspekte des Raums in den Blick genommen (vgl. Mania et al. 2015). So werden der Einfluss der Entfernung vom Wohnort zur Weiterbildungseinrichtung (vgl. Klaus-Roeder 1983; Feldmann und Hartkopf 2006; Feldmann und Schemmann 2008) oder die Bedeutung regionaler Gelegenheitsstrukturen von Angeboten der Erwachsenen- und Weiterbildung (vgl. Herbrechter et al. 2011; Martin et al. 2015, 2016; Sixt et al. 2018) untersucht. Der Raumbezug ist in früheren Studien eher implizit, sodass die Bedeutung einzelner Raumdimensionen untersucht wurde, ohne den Raumbegriff explizit theoretisch herzuleiten. Seit dem „Spatial Turn“ (vgl. Günzel 2009; Weidenhaus 2013) in den Kultur- und Sozialwissenschaften Ende der 1980er Jahre wird der Diskurs zum Raum und Sozialraum vermehrt und vor allem explizit unter der Raumperspektive geführt. Die symbolisch-institutionellen, materiell-infrastrukturellen und sozial-interaktiven Aspekte des Raums werden stärker verschränkt und damit die Relationalität des Begriffes im Anschluss an Löw (2001) berücksichtigt (vgl. Mania et al. 2015). Inzwischen gibt es eine Vielzahl von Publikationen, welche die möglichen Raumperspektiven verdeutlichen (vgl. Döring und Thielmann 2008; Dünne und Günzel 2006; Kessl et al. 2005). Raum wird nun als (weitere) „Kategorie herangezogen, mit der pädagogisch relevante Differenzstrukturen beobachtet werden können“ (Nugel 2014, S. 30). „Der Anschluss an die sozial- und kulturwissenschaftliche Raumforschung ist in der Erziehungswissenschaft noch nicht ganz geschafft, aber die nachholende Konjunktur der vergangenen Jahre macht Hoffnung", so Kessl (2016, S. 15).

Im Bereich der Sozialen Arbeit gibt es seit vielen Jahren eine Auseinandersetzung mit dem (Sozial-)Raum (vgl. Fritsche et al. 2010). Früchtel et al. (2010) haben ausgehend vom relationalen Raumbegriff ein umfassendes sozialräumliches Modell vorgelegt, das im SONI-Schema folgende Dimensionen berücksichtigt: Sozialstruktur, Organisation mit dem Fokus auf System, sowie Netzwerk und Individuum, mit dem Fokus auf Lebenswelt. Die Kombination der vier Dimensionen ermöglicht ein „Weitwinkelobjektiv einer komplexen Sicht auf komplexe Dinge“ (Budde und Früchtel 2011, S. 15). Die vier Dimensionen werden dabei als Handlungsfelder und Ebenen beschrieben, die durch die ,Verbindung von Fall, Feld, Organisation und Struktur" (Früchtel et al. 2010, S. 11) einen mehrdimensionalen Sozialen Raum eröffnen. Im Sinne des relationalen Raumbegriffs ermöglichen die vier Dimensionen nicht nur den Blick auf etwa physische Entfernungen, (Weiterbildungs-)Infrastruktur und Weiterbildungsorganisationen, sondern ebenfalls auf Beziehungen, Interaktionen, soziale Verhältnisse sowie die Interessen und Handlungen von Subjekten. Sozialraumorientierung versteht sich dabei als eine ,,unter Nutzung und Weiterentwicklung verschiedener theoretischer und methodischer Blickrichtungen entwickelte Perspektive“ (Hinte 2009, S. 23), die auch für eine erwachsenenbildnerische Theoriebildung genutzt werden kann. 


\section{Methodischer Hintergrund}

Im Folgenden wird auf die empirischen Daten eingegangen, die vor dem Hintergrund der Fragestellung des Beitrags neu interpretiert wurden. Die Daten stammen aus einer Studie zu Regulativen der Weiterbildungsbeteiligung sogenannter ,,bildungsferner Gruppen“, bei der als Ergebnis ein sozialräumliches Modell von Regulativen der (Nicht-)Teilnahme an organisierter Weiterbildung entwickelt wurde (vgl. Mania 2018).

Die Untersuchung fand in einem zunächst administrativ bestimmten Gebiet in Berlin statt, dem Quartier Soldiner-/Wollankstraße. Entsprechend der Diskussion um Sozialraum als „relationalen Raum“ (Löw 2001) waren jedoch nicht die administrativen Grenzen, sondern die Wahrnehmung von (Lern-)Orten entscheidend für den Feldzugang, sodass letztlich nicht die Bewohnerinnen und Bewohner des Quartiers, sondern die Besucherinnen und Besucher des Quartiers - unabhängig von ihrem Wohnort - befragt wurden. Der Zugang zu den insgesamt 49 Interviewten erfolgte über „Ankerpunkte“ (vgl. Kronauer 2014) im Quartier, d.h. über bekannte und vertraute Orte, wie Kindertagesstätten, Schulen, Vereine, soziale und religiöse Einrichtungen, Stadtteiltreffs, Weiterbildungseinrichtungen und Arbeitgeber. Im Sinne des Theoretical Samplings nach Strauss und Corbin (1996) wurde eine Kombination verschiedener Samplingstrategien genutzt, die je nach Forschungs- bzw. Auswertungsphase und Feldzugang variiert haben. Dabei ging es um eine bewusste Auswahl von Fällen, die während der Analyseaktivität eine besondere theoretische Bedeutung erhalten haben (vgl. Hülst 2010, S. 290). Um möglichst viele Variationen und Dimensionen in den Daten zu gewinnen, die Kategorien anzureichern und die theoretische Sättigung zu erreichen, wurden möglichst unterschiedliche Anlaufstellen ausgewählt. Aufgrund des spezifischen Feldzugangs finden sich in dem Sample vor allem Personen ohne oder mit einem niedrigen schulischen bzw. beruflichen Abschluss, die zum Zeitpunkt des Interviews arbeitssuchend oder nicht erwerbstätig waren. Das Alter lag zwischen 21 und 87 Jahren. Von den etwa zur Hälfte männlichen bzw. weiblichen Befragten verfügte die Mehrheit über einen Migrationshintergrund. Die meisten der 49 Befragten hatten Kind(er).

Die Datenerhebung erfolgte mittels problemzentrierter Interviews nach Witzel (2000, 1982). Dieses Verfahren zählt zu den halbstrukturierten Interviewformen (vgl. Krüger 1999) und kombiniert ,verschiedene Elemente einer leitfadenorientierten und teilweise offenen Befragung" (Witzel 1982, S. 67). Diese wurden um sozialraumorientierte Analysemethoden (vgl. Deinet 2009), wie den Einsatz einer „Stadtkarte“, ergänzt, die während des gesamten Interviews als Ausgangspunkt für Erzählstimuli und Fragen diente.

Die gegenstandbezogene Datenauswertung erfolgte in Anlehnung an die Grounded Theory von Strauss und Corbin (1996), wobei das sozialräumliche SONI-Schema (vgl. Früchtel et al. 2010) mit den Dimensionen Sozialstruktur, Organisation, Netzwerk und Individuum als Heuristik zur Systematisierung und Interpretation der empirischen Ergebnisse diente. Die identifizierten Regulative der Weiterbildungsbeteiligung wurden im Prozess der Auswertung entlang der vier sozialräumlichen Dimensionen zugeordnet. Die neunzehn Regulative wurden im Hinblick auf Ihre jeweiligen Aspekte und Facetten dargestellt und sind zudem nicht 
isoliert zu sehen, sondern auf vielfällige Weise miteinander verbunden, bedingen sich gegenseitig und wirken kumulativ.

Für diesen Beitrag wurde das empirische Material im Sinne einer Sekundärauswertung einer zeittheoretisch orientierten Analyse unterzogen, sodass die sozialräumlichen Regulative im Hinblick auf temporale Bezüge geprüft wurden. Als Ausgangspunkt wurde auf das Modell von Schmidt-Lauff (2018) zurückgegriffen, vor allem auf die Kategorie „Selbstverhältnisse zu Zeit“, welche die subjektive Sicht auf temporale Bezüge ausdrückt.

\section{Empirische Ergebnisse}

Die Darstellung der empirischen Ergebnisse erfolgt im Folgenden entlang der von Schmidt-Lauff (2018) vorgeschlagenen Kategorien. Bei der Darstellung der temporalen Bezüge wird dabei auf die Daten zu den sozialräumlichen Regulativen (vgl. Mania 2018) zurückgegriffen.

\subsection{Selbstverhältnis einer temporalen Dimensionsverschränkung}

Die temporale Dimensionsverschränkung bezieht sich auf die „Lerninitiativen und -gründe zwischen Vergangenheit, Gegenwart und Zukunft" (Schmidt-Lauff 2018, S. 332). Im Sinne des Prinzips der „Erfahrungsorientierung“ geht es beispielsweise um vergangene Lernerfahrungen, während kommende Ereignisse, wie neue Aufgaben oder drohender Arbeitsplatzverlust, an zukünftige Relevanzen und Bildungsaspirationen gebunden sind.

Die vergangenen Lernerfahrungen (Regulativ Bildungserfahrungen) werden in den Interviews zum einen auf die Schulzeit und zum anderen auf bisherige Weiterbildungserfahrungen bezogen. So wird die Ablehnung organisierter Weiterbildung mit negativen Erinnerungen an die eigene Schulzeit begründet (I 26, A. 612; I 3, A. 94). Negative Weiterbildungserfahrungen beruhen beispielsweise auf der Beurteilung der Weiterbildungsanbieter (I 40, A. 129), den Erfahrungen mit der Unterrichtsgestaltung durch die Lehrenden (I 45, A. 142-148) und dem fehlenden Nutzen (,Geldverschwendung und Zeitverschwendung“) (I 45, A. 158). Sie führen zur Ablehnung organisierter Weiterbildung. Sind die Erinnerungen an bisherige Lernprozesse in Bildungsinstitutionen dagegen positiver Art, so wird Weiterbildung als selbstverständlich wahrgenommen oder stellt jedenfalls eine Handlungsoption dar. Im Zuge der Teilnahme an einem bestimmten Angebot in einer Einrichtung stößt man - mehr oder weniger zufällig - auf weitere dort durchgeführte Angebote: „Das habe ich so hier mitbekommen" (I 15, A. 166). Überdies wird von den Lehrenden im Sinne eines Übergangsmanagements oder einer Übergangsberatung auf weitere passende Angebote aufmerksam gemacht (I 5, A. 160).

\footnotetext{
2 Die Interviews wurden mit einer Abkürzung anonymisiert: I= Interview, nach der Reihenfolge in der Interviewdurchführung durchnummeriert; $\mathrm{A}=$ die Absatznummer der Aussage im dazugehörigen InterviewTranskript der MAXQDA-Datei.
} 
Die Zukunftsorientierung des Lernens wird in den Interviews bei den Ausführungen zu den erwarteten Nutzendimensionen sichtbar (Regulativ Nutzenerwartungen). Anstehende Aufgaben und dafür benötigte Kompetenzen werden als Gründe für den Besuch einer Weiterbildung genannt (I 26, A. 30-31; I 21, A. 277). Als weiterer Aspekt der Zukunftsorientierung wird die berufliche Chancenverbesserung aufgeführt, die meist mit dem Erwerb eines Abschlusses einhergeht, der Voraussetzung für einen bestimmten Arbeitsplatz oder eine angestrebte Position ist (I 9; I 10, A. 94; I 28, A. 43; I 29, A. 234; I 34; I 25, A. 156; I 41; I 35; I 23; I 14; I 44). Die Teilnahme an abschlussbezogenen Veranstaltungen allgemeiner oder beruflicher Weiterbildung kann auch die Voraussetzung für weitere Qualifizierungsmaßnahmen oder Fortbildungen sein. In manchen Fällen entsteht überhaupt, ,die Chance, sich noch mehr weiterzubilden“ (I 42, A. 12), weil ein bestimmtes Zertifikat oder ein Abschluss erst die Zugangsvoraussetzung für weitere Weiterbildungsgänge bietet (I 7; I 43, A. 103; I 9, A. 70).

\subsection{Selbstverhältnis expliziter Zeitanteile}

Mit expliziten Zeitanteilen sind vor dem Hintergrund von ,Zeitknappheit und -konkurrenzen“"Zeitfenster für Bildung und Lernen gemeint, die von anderen Tätigkeiten „freigehalten und entlastet“ (Schmidt-Lauff 2018, S. 332) werden.

In den Interviews wird sowohl von ausgewiesenen Lernzeiten während der Arbeitszeit (Regulativ Arbeitsbezogene Gelegenheitsstrukturen) berichtet - womit in der Regel die innerbetrieblichen, während der Arbeitszeit stattfindenden Weiterbildungsveranstaltungen gemeint sind - als auch auf die ,Verwobenheit“ von Arbeit und Lernen bei selbstorganisierten Lernprozessen bzw. dem Lernen am Arbeitsplatz verweisen. Lernen erfolgt dabei oft „automatisch“ (I 35, A. 212) und „nebenbei“ (I 3, A. 122).

Die familiale Unterstützung wird als ein Faktor genannt, um ,freigehaltene Zeiten“ entstehen zu lassen. Durch die Übernahme und Aufteilung von Haushaltspflichten und Kinderbetreuung (I 6) können freie Zeiten für eine Weiterbildungsteilnahme ermöglicht werden (Regulativ Familiale Unterstützung).

$\mathrm{Zu}$ den „Zeitfenstern“ für den Besuch von Weiterbildungsveranstaltungen ist vor dem Hintergrund der empirischen Daten nicht nur die reine Anwesenheit in einem Lernangebot zu zählen, sondern auch die An- und Abreisezeit. Die Entscheidung für oder gegen eine Teilnahme an Weiterbildung wird vielfach von der Entfernung und der Erreichbarkeit des Veranstaltungsorts abhängig gemacht, wobei fußläufig (,,kann halt einfach laufen" [I 3, A. 48]) oder schnell erreichbare Strecken (I 32, A. 509; I 34, A. 338) als vorteilhaft empfunden werden und bei der Wahl zwischen mehreren Angeboten entscheidungsrelevant sind (I 32, A. 578) (Regulative Sozialräumliche Entfernungen sowie Grenzen und Mobilität).

\subsection{Selbstverhältnis der flexiblen Kontinuität}

Die individuelle Flexibilität für Lernen ist angesiedelt ,zwischen Arbeitszeit und Freizeit und externer Rahmung zur Rhythmisierung und Strukturierung“ (SchmidtLauff 2007, S. 232). 
Unter dieser Kategorie lassen sich die ,zeitlichen Strukturwünsche“ bestimmter Branchen oder Betriebe (Regulativ Arbeitsbezogene Gelegenheitsstrukturen) zusammenfassen, die z. B. vorschreiben, ,zu welcher Zeit und wie oft im Jahr Lernen stattfinden soll und mit welchem zeitlichen Umfang“ (Schmidt-Lauff 2018, S. 333). So können branchenspezifische Weiterbildungsverpflichtungen als Gelegenheitsstrukturen der Arbeitswelt gesehen werden, die die Beteiligung an Weiterbildung erhöhen bzw. ermöglichen. In einigen Branchen, z.B. den Bereichen Pflege und Medizin, in denen regelmäßige Weiterbildungen verpflichtend vorgesehen sind (I 44, A. 182; I 14; I 23, A. 149; I 30, A. 121; I 9; I 30; I 35). In den Interviews wird in diesem Kontext beispielsweise von monatlichen Vortragsreihen, die am Arbeitsplatz angeboten werden (I 21, A. 27; I 23, A. 149) oder von zwei verpflichtenden Fortbildungen pro Jahr berichtet (I 22, A. 182; I 21, A. 181).

Der Aspekt der Flexibilität wird in den Interviews im Hinblick auf die Angebotsformate der Weiterbildungsanbieter thematisiert (Regulativ Angebotsgestaltung). Die terminliche Gestaltung der Angebote sei „oft so starr und so eng“ (I 14, A. 199), sodass informelle bzw. selbstorganisierte Lernformen bevorzugt werden.

\subsection{Selbstverhältnis temporaler Formalisierung}

Ausgehend von den bereits beschriebenen ,temporalen Anforderungen an Lernzeiten“ (Schmidt-Lauff 2018, S. 333) geht es nun um „Formalisierungen von Lernen zwischen (De-)Institutionalisierung und Selbststeuerung als temporale Gegentendenz" (Schmidt-Lauff 2007, S. 232). Temporale Strukturgeber sind solche ,zeitlichen Maßstäbe wie Seriation, Häufigkeit, Dauer, Synchronisation“ (Schmidt-Lauff 2018, S. 333).

Im Hinblick auf die genannten Maßstäbe ist beispielsweise das Vorhandensein einer „Probestunde“ (Regulativ Zugangsportale) förderlich für die Weiterbildungsbeteiligung, da so ein niedrigschwelliger Zugang entsteht, indem die Adressatinnen und Adressaten ein Angebot erstmal (vor-)testen können, ohne sich direkt verbindlich anmelden zu müssen. Gerade bei Weiterbildungsangeboten von längerer Dauer spielt diese Möglichkeit eine Rolle, da noch keine dauerhafte terminliche Verpflichtung notwendig wird, ohne das Angebot zu kennen (I 14, A. 229).

Bezieht man die temporalen Anforderungen auf Kurszeiten und -termine (Regulativ Angebotsgestaltung) seitens der Weiterbildungsorganisationen, geht es in erster Linie darum, diese passend $\mathrm{zu}$ den Lebenswelten und Routinen der potentiellen Teilnehmenden zu gestalten. So werden in den Interviews passende Anfangszeiten von Weiterbildungsangeboten als Teilnahmebedingung aufgeführt. Eine Teilnahme an Weiterbildung wird bspw. davon abhängig gemacht, ob es vormittags stattfindet: „Aber ich möchte wenn dann tagsüber, weil er ist tagsüber in der Schule. Im Kindergarten, sie in der Schule“ (I 48, A. 199). Neben der zeitlichen Terminierung der Weiterbildungsveranstaltung sind auch die Anzahl der Termine und die Regelmäßigkeit der Teilnahme relevant (I 21; I 14, A. 227; I 34, A. 334). Oft wird in den Interviews die Teilnahme an institutionalisierter Weiterbildung davon abhängig gemacht, ob flexible oder ,kürzere“ (I 39, A. 198) Lernformate angeboten werden. 


\subsection{Selbstverhältnis struktureller und biografischer Zeitkonkurrenzen}

Angesichts des Zeitaufwands und im Kontext auszubalancierender Zeitkonkurrenzen muss für das Lernen eine Entscheidung getroffen werden. So steht Lernen in ständigem Wettbewerb zu anderen Tätigkeiten, die sowohl von strukturellen Faktoren, wie Alter oder Geschlecht, als auch von den jeweiligen Lebensphasen, wie Familiengründung, beruflicher Aufstieg oder Eintritt ins Rentenalter, abhängig sind (vgl. Schmidt-Lauff 2018, S. 334).

Der strukturelle Faktor Alter wird in den Interviews vor allem hinsichtlich seines Einflusses auf den erwarteten bzw. unterstellten Nutzen einer Weiterbildung (Regulativ Nutzenerwartungen) thematisiert: „Weiterbildung im Alter lohne sich nicht mehr" (I 12, A. 242). Geschlechtstypische Rollenbilder als kulturell vermittelte Vorstellungen zur Aufgabenverteilung zwischen Mann und Frau haben Einfluss auf den Stellenwert von (Weiter-)Bildung in bestimmten Lebensphasen (Regulative Kapitalausstattung sowie Nutzenerwartungen) und können dem strukturellen Faktor Geschlecht zugeordnet werden. Traditionelle Rollenvorstellungen der Frau als „Hausfrau“, führen dazu, dass (berufliche) Weiterbildung keine oder nur geringe Bedeutung einnimmt. Dadurch, dass die Frau ,immer Zuhause“ (I 11, A. 769) ist und die Rolle der „Hausfrau“ übernimmt, geraten berufliche Nutzenerwartungen zeitweise oder für das gesamte restliche Leben in den Hintergrund (auch I 17, A. 152; I 18, A. 75): „Na ja, früher hat man ja ... war man ja mehr zu Hause. (...) Die Hausfrau war halt man ja“ (I 16, A. 146).

Die Beteiligung an organisierter Weiterbildung spielt in bestimmten Lebensphasen, d.h. unter bestimmten privaten oder beruflichen Umständen, keine oder eine nur geringe Rolle, da andere Aktivitäten und Aufgaben bevorzugt werden. So gibt es Umbrüche und Statuswechsel im Lebenslauf oder biografische Übergänge, wie Familiengründung, Trennung, Migration, Krankheit, Berufswechsel, (Langzeit-)Arbeitslosigkeit und Ruhestand (Regulativ Kritische Lebensereignisse), die eine Veränderung in Bezug auf die Beteiligung an Weiterbildungsaktivitäten mit sich bringen. Kritische Lebensereignisse können Lernanlässe schaffen und Bildungsinteresse wecken, verstärken und festigen, aber auch die Weiterbildungsteilnahme negativ beeinflussen, da der Besuch eines Bildungsangebots durch die Veränderung der Lebensumstände weniger relevant erscheint.

Beispielhaft sei hier das Ereignis Familiengründung herangezogen. In den Interviews zeigt sich vor allem bei Müttern eine starke Orientierung an den Interessen des Kindes, vor allem in seinen ersten Lebensjahren: „Eigentlich ist es nur das Kind. Und der Rest ist eher so hinten ran gestellt" (I 3, A. 124). Der gesamte Tagesablauf richten sich nach dem Tagesrhythmus des Kindes, sodass Aktivitäten im Vordergrund stehen, an denen das Kind alleine oder mit dem Elternteil teilnimmt (I 10, A. I 40; I 15, A. 126, 308, 616; I 48; I 49; I 45). Familiäre Verpflichtungen scheinen in dem Kontext einerseits eine Weiterbildungsbarriere zu sein. Andererseits führt die Elternschaft zu Entstehung neuer Bildungsinteressen und der Teilnahme an Angeboten im Bereich Familienbildung. Es handelt sich dabei um eine bewusste Fokussierung auf die Bedürfnisse des Kindes und eine zeitlich begrenzte Phase im Lebenslauf, die sich als Familienphase zusammenfassen lässt (I 48, A. 177; I 9, A. 80-84). 


\subsection{Selbstverhältnis einer Ökonomisierung von Lernzeit}

Die Ökonomisierung von Lernzeit bezieht sich auf eine ,zukünftige Verwertungsausrichtung und effizienzbetonte Prozessgestaltung“ (Schmidt-Lauff 2018, S. 334) des Lernens.

Unter diesen Gesichtspunkten wird Lernen nur in seiner Verwertungsfunktion (Regulativ Nutzenerwartungen) gesehen, sodass der Prozess des Lernens selbst vernachlässigt und ignoriert wird. In den Interviews zeigt sich dies insbesondere in den folgenden ,Wenn-dann-Formulierungen“:

- „Wenn wir das brauchen, dann machen wir das auch“ (I 9, A. 72).

- „Wenn ich es bräuchte, dann würde ich es natürlich machen“ (I 22, A. 471).

Außerdem geht die Ökonomisierung von Lernzeit mit einer Reduzierung von Lernzeit zu einem „reinen Kostenfaktor“ einher, die sich in den „Kosten-NutzenAbwägungen“ der Interviewten belegen lassen. Fällt die Kosten-Nutzen-Abwägung so aus, dass der Aufwand als vertretbar oder gering eingeschätzt wird, kommt eine Weiterbildungsteilnahme in Frage (I 26, A. 24). Zudem wird die Dauer einer Weiterbildung zu einem entscheidungsrelevanten Faktor der für die Teilnahme oder NichtTeilnahme: ,[S]echs Wochen, da kann man nicht so viel falsch machen. (...) Und dann habe ich das gemacht" (I 34, A. 106-120). Bevorzugt werden Angebote die „kurz“ (I 39, A. 198-200) sind und ,nicht lange dauern“ (I 34, A. 130-132).

\subsection{Selbstverhältnis temporal divergenter Strukturen}

Diese Kategorie umfasst das Verhältnis von „Bildung und Lernen zwischen ökonomischen und pädagogischen Zeitordnungen und -strukturen“ (Schmidt-Lauff 2007, S. 233).

Die Ausdehnung des Lernens über die Lebenszeit kann unterschiedlich bewertet werden: als Chance, als äußerer Druck oder als Selbstverständlichkeit. In den Interviewdaten lassen sich alle drei Bewertungsmuster wiederfinden.

So wird lebenslanges Lernen als Möglichkeit der ,,persönlichen Weiterentwicklung“ gesehen (Regulativ Nutzenerwartungen). Die Teilnahme an Weiterbildungsveranstaltungen wird mit allgemeinem Interesse am Lernen begründet, wobei Bildung als Persönlichkeitsentwicklung verstanden wird: „Ja, weil, ich wollte lernen. Ich wollte nie, ich, also ich sag immer, verblöden“ (I 23, A. 100). Neues zu lernen und neue Fähigkeiten zu erlangen sei ,für die Seele sehr gesund“ (I 24, A. 280) und ein Weg der „Eigenverwirklichung“ (I 34, A. 314).

„Lebenslang lernen“ kann als äußerer Druck empfunden werden, wenn dies von den Vorgesetzten erwartet wird oder die Weiterbildung explizit für die Angestellten angewiesen wird (Regulativ Arbeitsbezogene Gelegenheitsstrukturen). So wird von den Interviewten berichtet, dass sie eine berufliche Fortbildung oder Qualifizierung aufgrund der individuellen Ansprache durch einen Vorgesetzten wahrgenommen haben (I 40, A. 159; I 28, A. 61). Hierzu ein beispielhaftes Zitat: ,Von mir aus hätte ich keine Kurse gemacht. Ich war mit dem zufrieden mit dem was ich hatte. Mit der Arbeit“ (I 40, A. 159). 
Lernen über die Lebenszeit als Selbstverständlichkeit zeigt sich dagegen in folgenden Aussagen: „Man muss ja immer weitermachen. Man muss ja immer dazu lernen im Leben“ (I 43, A. 132).

\subsection{Selbstverhältnis von Zeit als Fluchtkategorie}

Die Kategorie verweist auf die „Vielschichtigkeit zeitlicher Strukturen und Erfahrungen“" und die „Nichtkommunizierbarkeit temporaler Eindrücke“ (Schmidt-Lauff 2018, S. 331). Damit ist gemeint, dass sich hinter der Aussage „Ich habe keine Zeit" oft nicht-temporale Aspekte verbergen, wie Übernahme von Verantwortung für Familie bzw. Beruf sowie Bedürfnisse nach Ruhe, Erholung und Spaß.

In den Interviews lässt sich dies belegen, da auf die Aussage „Ich habe keine Zeit“" verschiedene weitere Begründungsmuster folgen (Regulativ Ressourcen): Als ein Grund für fehlende zeitlichen Ressourcen für Weiterbildung werden oft Kinder und die damit verbundenen Aufgaben genannt: ,Weil ich habe keine Zeit durch Kinder“ (I 29, A. 16). In vielen Interviews wird die erforderliche Arbeit im Haushalt als Grund für Nicht-Teilnahme an Weiterbildung in der Vergangenheit oder Zukunft genannt. Dabei fallen in den Interviews folgende typische Aussagen: „Also, ich habe eigentlich auch nicht so viel Zeit, weil ich ja den ganzen Tag mit dem Haushalt" (I 15, A. 204). Als eine weitere zeitraubende Aufgabe wird die Pflege von Angehörigen beschrieben (I 43, A. 74; I 6, A. 134). Temporale Aspekte finden sich auch in der Thematisierung von Ruhebedürfnissen wieder, die oft im Zusammenhang mit tätigkeitbezogenen Merkmalen, wie lange Arbeitszeiten, thematisiert werden (I 26, A. 61; I 21, A. 433). Meist wird argumentiert, dass eine Teilnahme an Weiterbildungsveranstaltungen nach der Arbeit nicht mehr infrage kommt, da man sich nach der Arbeit nur noch ,ausruhen“ (I 28, A. 81) wolle und keine Kraft mehr für Lernaktivitäten habe: „Wenn ich nach Hause komme bin ich platt“ (I 22, A. 477). Zudem wird auf die Herausforderung der Vereinbarkeit von Familie und Beruf verwiesen (I 21, A. 448). Die Analyse der Interviews hat ergeben, dass mangelnde Zeitressourcen vor allem von den Interviewten genannt werden, die von der Beteiligung an Weiterbildung keinen Nutzen erwarten. Insbesondere bei Angeboten im Bereich Sport, Schwimmen oder Tanz wird fehlende Zeit als Argument eingebracht. Andererseits werden niedrigschwellige Lernangebote vor Ort in Anspruch genommen, von deren Teilnahme man sich einen konkreten (praktischen) Nutzen verspricht, z. B. der Erwerb von Kompetenzen in der Kindererziehung. Fehlende Nutzenerwartungen zeigen sich oft durch die Bevorzugung von Freizeitaktivitäten und der Ausübung von Hobbys. Aktivitäten wie Sport (I 39, A 155), Kochen (I 30), Dekorieren/Basteln (I 29), Gartenarbeit (I 31, A. 840), Tierhaltung (I 33, A. 288; I 36, A. 330) und Handwerken (I 45) sind im Vergleich zur Teilnahme an Weiterbildung prioritär.

\section{Diskussion und Ausblick}

Die Ergebnisse der zeittheoretisch orientierten Analyse belegen, dass die Regulative der (Nicht-)Teilnahme an organisierter Weiterbildung entlang der vier sozialräumli- 
Tab. 1 Überblick über Verbindungen zwischen sozialräumlichen Regulativen und Zeitkategorien

\begin{tabular}{|c|c|c|}
\hline $\begin{array}{l}\text { Raum } \\
\text { „Regulative“ }\end{array}$ & & $\begin{array}{l}\text { Zeit } \\
\text { „Selbstverhältnisse“ }\end{array}$ \\
\hline \multirow[t]{3}{*}{$\overline{\text { Sozialstruktur }}$} & $\begin{array}{l}\text { Sozialräumliche Entfernungen und } \\
\text { Grenzen: } \\
\text { Entfernungen im Sozialraum }\end{array}$ & Selbstverhältnis expliziter Zeitanteile \\
\hline & $\begin{array}{l}\text { Arbeitsbezogene Gelegenheitsstruk- } \\
\text { turen: } \\
\text { Gelegenheitsstrukturen der Arbeits- } \\
\text { welt }\end{array}$ & $\begin{array}{l}\text { Selbstverhältnis temporal divergenter Struktu- } \\
\text { ren } \\
\text { Selbstverhältnis der flexiblen Kontinuität } \\
\text { Selbstverhältnis expliziter Zeitanteile }\end{array}$ \\
\hline & $\begin{array}{l}\text { Kapitalausstattung: } \\
\text { kulturelles Kapital }\end{array}$ & $\begin{array}{l}\text { Selbstverhältnis struktureller und biografi- } \\
\text { scher Zeitkonkurrenzen }\end{array}$ \\
\hline \multirow[t]{2}{*}{ Organisation } & $\begin{array}{l}\text { Zugangsportale: } \\
\text { Probestunde; Veranstaltungsorte und } \\
\text { Vernetzung }\end{array}$ & Selbstverhältnis temporaler Formalisierung \\
\hline & $\begin{array}{l}\text { Angebotsgestaltung: } \\
\text { Kurszeiten und -termine; Kinderbe- } \\
\text { treuung }\end{array}$ & $\begin{array}{l}\text { Selbstverhältnis temporaler Formalisierung } \\
\text { Selbstverhältnis der flexiblen Kontinuität }\end{array}$ \\
\hline Netzwerk & Familiale Unterstützung & Selbstverhältnis expliziter Zeitanteile \\
\hline \multirow[t]{6}{*}{ Individuum } & Mobilität & Selbstverhältnis expliziter Zeitanteile \\
\hline & Kritische Lebensereignisse & $\begin{array}{l}\text { Selbstverhältnis struktureller und biografi- } \\
\text { scher Zeitkonkurrenzen }\end{array}$ \\
\hline & Bildungserfahrungen & $\begin{array}{l}\text { Selbstverhältnis einer temporalen Dimensi- } \\
\text { onsverschränkung }\end{array}$ \\
\hline & $\begin{array}{l}\text { Lernstrategien und -vorstellungen: } \\
\text { Bevorzugung anderer Lernformen }\end{array}$ & Selbstverhältnis expliziter Zeitanteile \\
\hline & $\begin{array}{l}\text { Nutzenerwartungen: } \\
\text { Einflussfaktoren der Nutzenerwar- } \\
\text { tung; Nutendimensionen }\end{array}$ & $\begin{array}{l}\text { Selbstverhältnis einer Ökonomisierung von } \\
\text { Lernzeit } \\
\text { Selbstverhältnis temporal divergenter Struktu- } \\
\text { ren } \\
\text { Selbstverhältnis struktureller und biografi- } \\
\text { scher Zeitkonkurrenzen } \\
\text { Selbstverhältnis einer temporalen Dimensi- } \\
\text { onsverschränkung } \\
\text { Selbstverhältnis von Zeit als Fluchtkategorie }\end{array}$ \\
\hline & $\begin{array}{l}\text { Ressourcen: } \\
\text { Zeit }\end{array}$ & Selbstverhältnis von Zeit als Fluchtkategorie \\
\hline
\end{tabular}

chen Dimensionen - Sozialstruktur, Organisation, Netzwerk und Individuum - vielfältige temporale Bezüge aufweisen (vgl. Tab. 1). Die in der Heuristik von SchmidtLauff (2018) identifizierten „Selbstverhältnisse zu Zeit“ lassen sich alle in den empirischen Daten wiederfinden und verdeutlichen die Vielschichtigkeit der Kategorie Zeit.

So zeigen sich beispielsweise mit Blick auf die Regulative „Sozialräumliche Entfernungen und Grenzen“, „Arbeitsbezogene Gelegenheitsstrukturen“, „Angebotsgestaltung“, „Familiale Unterstützung“, „Kritische Lebensereignisse“, „Bildungserfahrungen“ sowie „Nutzendimensionen“ vielfältige temporale Implikationen und zeittheoretische Bezüge. Zeit erscheint dabei in unterschiedlichen Bedeutungen als „passender“ Zeitpunkt, als Dauer, als Überwindung einer Entfernung, als Prioritätensetzung in verschiedenen Lebensphasen und -situationen (Zeitkonkurrenzen), als 
Aufwand und Kostenfaktor und im Verhältnis zu vergangenen Bildungserfahrungen und zukünftigen Aktivitäten, um nur wenige Aspekte exemplarisch zu nennen. Ein sozialräumliches Regulativ kann dabei mehrere temporale Bezüge beinhalten, genauso wie ein bestimmtes „Selbstverhältnis zu Zeit“ sich in mehreren Regulativen finden lässt, was die Verwobenheit der beiden Kategorien Zeit und Raum verdeutlicht. Nicht nur zwischen den einzelnen Regulativen der Weiterbildungsbeteiligung gibt es daher Verbindungen, sondern auch die Kategorien Zeit und Raum sind miteinander verschränkt.

Die Interviewdaten verdeutlichen, welche Begründungen hinter der Aussage ,,keine Zeit für Weiterbildung“" stehen können und wie die Individuen über Zeitkonkurrenzen und Zeitpräferenzen in verschiedenen Lebensphasen, in Abhängigkeit von aktueller Lebenslage und kritischer Lebensereignisse wie Elternschaft sprechen. Diese Ergebnisse können als Weiterführung der empirischen Arbeiten von Schmidt-Lauff (2018) gesehen werden, sind aber auch im Rahmen der Forschung zu Weiterbildungsbeteiligung nutzbar. Vor allem für standardisierte Erhebungen zu Weiterbildungsbarrieren stellt sich die Frage, inwiefern die Kategorie „keine Zeit““ weiterzuführen ist oder eher in andere Aspekte aufgelöst werden sollte, die noch zu operationalisieren sind. So könnten die hinter der Aussage ,keine Zeit“ stehenden Begründungen, Prioritätensetzungen, Abwägungen und Entscheidungen erfragt werden, die dann möglicherweise mehr Erklärungskraft für das Zustandekommen der Weiterbildungsaktivitäten besitzen.

Derzeit stellt Zeit nicht nur eine ,sozial akzeptierte“ Fluchtkategorie der Befragten dar, sondern scheint auch als ein von den Forschern häufig genutztes „Fluchtitem“ $\mathrm{zu}$ fungieren, ohne dass dieses theoretisch hergeleitet wird und seine Implikationen mitbedacht werden. Im Anschluss an Kuwan (2011, S. 389) könnten Items weniger allgemein formuliert werden, sondern sich mehr ,auf spezifische Lebenssituationen und auf konkretes Verhalten" beziehen. Zudem stellt sich die Frage, inwiefern komplexe, mehrdimensionale Modelle der Weiterbildungsbeteiligung wie das von Boeren et al. (2010) operationalisiert und überprüft werden könnten.

Hinsichtlich der Nutzung der Erkenntnisse der Weiterbildungsbeteiligungsforschung für die Programm- und Angebotsentwicklung (vgl. Fleige et al. 2018) in der Erwachsenen- und Weiterbildung scheinen Alternativen für die Antwortmöglichkeit „keine Zeit“ sinnvoll, um so mögliche relevante Aspekte auf der Seite der Weiterbildungsanbieter stärker in den Blick zu nehmen (vgl. Robak et al. 2015). Statt der Fokussierung auf Zeitmangel als Grund für die Nicht-Teilnahme an Weiterbildungsangeboten wäre die stärkere Berücksichtigung von einrichtungs- und angebotsbezogenen Barrieren wünschenswert, die dann im Rahmen der Programmplanung berücksichtigt werden könnten. Dabei ist der Zusammenhang von Zeit, Raum und den Nutzenvorstellungen bzw. -erwartungen der Adressatinnen und -adressaten von Interesse, die zu den für Teilnahmeentscheidungen zunehmend relevanten Faktoren der Weiterbildungsbeteiligung zählen (vgl. Fleige 2015, 2011), worauf auch einige der o.g. Befunde hinweisen.

Grundsätzlich sind Lernen und Bildung immer in Zeit und Raum eingebunden. Beide Kategorien sind sehr vielschichtig, multidimensional und komplex, werden jedoch oft reduziert auf einzelne - leicht messbare - Aspekte. Die Diskurse zu Zeit 
und Raum - und mitunter auch zu Regulativen der Weiterbildungsbeteiligung werden bisher separat und isoliert geführt.

Die Ergebnisse der Analyse weisen darauf hin, dass eine sozialraumorientierte Datenerhebung nicht nur im Rahmen der Identifikation und Modellierung von Regulativen der Weiterbildungsbeteiligung fruchtbar, sondern auch geeignet ist, die Komplexität der Kategorie Zeit zu erkennen. Ein Zugang über Ankerpunkte in einem Sozialraum und die Nutzung von Stadtkarten als Erzählstimuli und Gesprächsanker bieten das Potenzial, gerade in Interviews mit sogenannten ,,bildungsfernen Gruppen“ die Befragten zu aktivieren und zu animieren, über Ihre Lern- und Bildungsprozesse zu sprechen und so das Problem der Verbalisierbarkeit zu reduzieren.

Die Gegenüberstellung der empirischen Ergebnisse zu den Regulativen der Weiterbildungsteilnahme und der acht Selbstverhältnisse zu Zeit verdeutlicht die Vielzahl der Verbindungen zwischen Zeit und Raum, ohne einzelne temporale Bezüge oder sozialräumliche Regulative in den Fokus zu rücken und detailliert zu beschreiben. Damit sollten die Vielschichtigkeit und Verwobenheit der Kategorien Raum und Zeit erst einmal aufgezeigt werden. Gleichzeitig war die Analyse nicht auf Vollständigkeit ausgelegt und zielte nicht auf das Finden aller temporalen Bezüge bei allen sozialräumlichen Regulativen, sondern darauf, die Bedeutung temporaler Bezüge in den Daten grundsätzlich zu belegen. Bei den genannten Regulativen handelt es sich daher um eine Auswahl, sodass weitere - sowohl explizite als auch implizite zeittheoretische Bezüge in den Daten denkbar sind. Vor dem Hintergrund der Forderung nach Integration von theoretischen Linien und integrierenden Ansätzen wäre eine Fokussierung der wechselseitigen Bedingtheit der Raum-Zeit-Beziehungen als nächster Analyseschritt wünschenswert.

Open Access Dieser Artikel wird unter der Creative Commons Namensnennung 4.0 International Lizenz (http://creativecommons.org/licenses/by/4.0/deed.de) veröffentlicht, welche die Nutzung, Vervielfältigung, Bearbeitung, Verbreitung und Wiedergabe in jeglichem Medium und Format erlaubt, sofern Sie den/die ursprünglichen Autor(en) und die Quelle ordnungsgemäß nennen, einen Link zur Creative Commons Lizenz beifügen und angeben, ob Änderungen vorgenommen wurden.

\section{Literatur}

Baethge, M., \& Baethge-Kinsky, V. (2004). Der ungleiche Kampf um das lebenslange Lernen. Münster: Waxmann.

Barz, H., \& Tippelt, R. (Hrsg.). (2004). Praxishandbuch Milieumarketing. Weiterbildung und soziale Milieus in Deutschland, Bd. 1. Bielefeld: W. Bertelsmann.

Bernhard, C. (2014). Region ungleich Region. Vom normativen Regionsbegriff zur interpretativen Regionalität. Report: Zeitschrift für Weiterbildungsforschung, 37(4), 83-95.

Bernhard, C., Kraus, K., Schreiber-Barsch, S., \& Stang, R. (Hrsg.). (2015). Erwachsenenbildung und Raum. Theoretische Perspektiven - professionelles Handeln - Rahmungen des Lernens. Bielefeld: W. Bertelsmann.

Bilger, F., \& Käpplinger, B. (2017). Barrieren für die Bildungsbeteiligung Erwachsener. In F. Bilger, F. Behringer, H. Kuper \& J. Schrader (Hrsg.), Weiterbildungsverhalten in Deutschland 2016. Ergebnisse des Adult Education Survey (AES) (S. 265-275). Bielefeld: W. Bertelsmann.

Bilger, F., \& Kuper, H. (2013). Zeit für Weiterbildung. In F. Bilger, D. Gnahs, J. Hartmann \& H. Kuper (Hrsg.), Weiterbildungsverhalten in Deutschland. Resultate des Adult Education Survey 2012 (S. 50-59). Bielefeld: W. Bertelsmann.

Bleckmann, P., \& Schmidt, V. (Hrsg.). (2011). Bildungslandschaften. Mehr Chancen für alle. Wiesbaden: Springer VS. 
Boeren, E., Nicaise, I., \& Baert, H. (2010). Theoretical models of participation in adult education: the need for an integrated model. International Journal of Lifelong Education, 1(29), 45-61. https://doi.org/ $10.1080 / 02601370903471270$.

Bolder, A. (2011). Das lebenslange Lernen, die Beteiligung daran und die Bildungspolitik. Und das lebenslange Lernen, die Beteiligung. In D. Holzer, B. Schröttner \& A. Sprung (Hrsg.), Reflexionen und Perspektiven der Weiterbildungsforschung (S. 53-66). Münster/München/Berlin: Waxmann.

Bremer, H., Kleemann-Göhring, M., \& Wagner, F. (2015). Weiterbildung und Weiterbildungsberatung für Bildungsferne. Ergebnisse aus der wissenschaftlichen Begleitung von Praxisprojekten in NRW. Bielefeld: W. Bertelsmann.

Brödel, R., \& Yendell, A. (2008). Weiterbildungsverhalten und Eigenressourcen. NRW-Studie über Geld, Zeit und Erträge beim lebenslangen Lernen. Bielefeld: W. Bertelsmann.

Brödel, R., Buschmeyer, H., Jütting, D. H., \& Niggemann, W. (1982). Bildungsurlaub in Nordrhein-Westfalen - Forschungsbericht der wissenschaftlichen Begleitung zum Modellprogramm Bildungsurlaub. Düsseldorf: Landeszentrale für politische Bildung.

Brüning, G., \& Kuwan, H. (Hrsg.). (2002). Benachteiligte und Bildungsferne - Empfehlungen für die Weiterbildung. Bielefeld: W. Bertelsmann.

Budde, W., \& Früchtel, F. (2011). Die Zukunft der Sozialraumorientierung: Theorie, Praxis und der Stand der Dinge. Evangelische Jugendhilfe, 88(1), 14-24.

Deinet, U. (2009). Analyse- und Beteiligungsmethoden. In U. Deinet (Hrsg.), Methodenbuch Sozialraum (S. 65-86). Wiesbaden: Springer VS.

Döring, J., \& Thielmann, T. (Hrsg.). (2008). Spatial Turn. Das Raumparadigma in den Kultur- und Sozialwissenschaften. Bielefeld: Transcript.

Dörpinghaus, A. (2008). Schonräume der Langsamkeit. Grundzüge einer temporalphänomenologischen Erwachsenenpädagogik. DIE-Zeitschrift für Erwachsenenbildung, 15(1), 42-45. https://doi.org/10. 3278/DIE0801W042.

Dünne, J., \& Günzel, S. (Hrsg.). (2006). Raumtheorie. Grundlagentexte aus Philosophie und Kulturwissenschaften. Frankfurt am Main: Suhrkamp.

Ebner von Eschenbach, M., \& Ludwig, J. (2015). Relationaler Raum und soziale Positionierung. Eine epistemoligische Reflexion zu Bildung. In C. Bernhard, K. Kraus, S. Schreiber-Barsch \& R. Stang (Hrsg.), Erwachsenenbildung und Raum. Theoretische Perspektiven - professionelles Handeln - Rahmungen des Lernens (S. 67-77). Bielefeld: W. Bertelsmann.

Faulstich, P. (2001). Zeitstrukturen und Weiterbildungsprobleme. In R. Dobischat \& H. Seifert (Hrsg.), Lernzeiten neu organisieren. Lebenslanges Lernen durch Integration von Bildung und Arbeit (S. 33-59). Berlin: Edition Sigma.

Feldmann, H. \& Hartkopf, E. (2006). Lernstandorte in raumsoziologischer Perspektive. DIE Zeitschrift. https://www.diezeitschrift.de/42006/feldmann06_01.htm. Zugegriffen: 19.06.2019.

Feldmann, H., \& Schemmann, M. (2008). Analyse von Weiterbildungsbeteiligungsstrukturen in lokalen Räumen - das Beispiel Bochum. In A. Grotlüschen (Hrsg.), Zukunft lebenslangen Lernens. Strategisches Bildungsmonitoring am Beispiel Bremens (S. 227-240). Bielefeld: W. Bertelsmann.

Fleige, M. (2011). Kulturen der Nutzenpositionierung. Durch Wissen zu veränderten Lernentscheidungen. Report. Zeitschrift für Weiterbildungsforschung, 3(34), 72-81.

Fleige, M. (2015). Nutzenvorstellungen von Teilnehmenden in beruflichen und erwerbsbiographischen Übergangssituationen. Befunde aus zwei Untersuchungen. In S. Schmidt-Lauff, H. v. Felden \& H. Pätzold (Hrsg.), Transitionen in der Erwachsenenbildung. Gesellschaftliche, institutionelle und individuelle Übergänge (S. 189-200). Opladen: Budrich.

Fleige, M., Gieseke, W., von Hippel, A., Käpplinger, B., \& Robak, S. (2018). Programm- und Angebotsentwicklung. In der Erwachsenen- und Weiterbildung. Bielefeld: W. Bertelsmann.

Fritsche, C., Lingg, E., \& Reutlinger, C. (2010). Raumwissenschaftliche Basics - eine Einleitung. In C. Reutlinger, C. Fritsche \& E. Lingg (Hrsg.), Raumwissenschaftliche Basics. Eine Einführung für die soziale Arbeit (S. 11-24). Wiesbaden: Springer VS.

Früchtel, F., Cyprian, G., \& Budde, W. (2010). Sozialer Raum und Soziale Arbeit. Textbook: theoretische Grundlagen (2. Aufl.). Wiesbaden: Springer VS.

Glaser, E., Koller, H.-C., Thole, W., \& Krumme, S. (Hrsg.). (2018). Räume für Bildung - Räume der Bildung. Beiträge zum 25. Kongress der Deutschen Gesellschaft für Erziehungswissenschaft. Opladen: Budrich.

Grotlüschen, A. (2010). Erneuerung der Interessetheorie. Die Genese von Interesse an Erwachsenen- und Weiterbildung. Wiesbaden: Springer VS.

Günzel, S. (2009). Einleitung. In S. Günzel (Hrsg.), Raumwissenschaften (S. 7-13). Frankfurt am Main: Suhrkamp. 
Herbrechter, D., Loreit, F., \& Schemmann, M. (2011). (Un-)Gleichheit in der Weiterbildung unter regionalen Vorzeichen. DIE - Zeitschrift für Erwachsenenbildung, 18(2), 27-30.

Hinte, W. (2009). Eigensinn und Lebensraum - zum Stand der Diskussion um das Fachkonzept ,Sozialraumorientierung“. Vierteljahresschrift für Heilpädagogik und ihre Nachbargebiete (VHN), 78(1), 20-33.

Hof, C. (2014). Erwachsenenpädagogische Dimensionen des Sozialraum: Eine Spurensuche. Erwachsenenbildung. Vierteljahresschrift für Theorie und Praxis, 60(3), 6-9.

Holzer, D. (2004). Widerstand gegen Weiterbildung. Weiterbildungsabstinenz und die Forderung nach lebenslangem Lernen. Wien: LIT.

Hülst, D. (2010). Grounded theory. In B. Friebertshäuser, A. Langer \& A. Prengel (Hrsg.), Handbuch qualitative Forschungsmethoden in der Erziehungswissenschaft (3. Aufl. S. 281-300). Weinheim: Juventa.

Kaufmann, K., \& Widany, S. (2013). Berufliche Weiterbildung - Gelegenheits- und Teilnahmestrukturen. Zeitschrift für Erziehungswissenschaft, 16(1), 29-54.

Kessl, F. (2016). Erziehungswissenschaftliche Forschung zu Raum und Räumlichkeit. Eine Verortung des Thementeils „Raum und Räumlichkeit in der erziehungswissenschaftlicher Forschung“. Zeitschrift für Pädagogik, 62(1), 5-19.

Kessl, F., \& Reutlinger, C. (2009). Zur materielen Relationalität des Sozialraums - einige raumtheoretische Hinweise. In A. Mörchen \& M. Tolksdorf (Hrsg.), Lernort Gemeinde. Ein neues Format der Erwachsenenbildung (S. 195-204). Bielefeld: W. Bertelsmann.

Kessl, F., Reutlinger, C., Maurer, S., \& Frey, O. (Hrsg.). (2005). Handbuch Sozialraum. Wiesbaden: Springer VS.

Klaus-Roeder, R. (1983). Sozialräumliche Strukturen und Weiterbildung. Am Beispiel der Volkshochschulen in Hessen. Baden-Baden: Nomos.

Kronauer, M. (2014). Inklusion und Exklusion in der Erwachsenenbildung. Der pädagogische Blick, 22(2), $126-135$.

Krüger, H.-H. (1999). Einführung in Theorien und Methoden der Erziehungswissenschaft (2. Aufl.). Opladen: Leske und Budrich.

Kuwan, H. (2011). Weiterbildungsbarrieren: Messung, empirische Befunde für Ältere und Schlussfolgerungen. In T. Eckert, A. von Hippel, M. Pietraß \& B. Schmidt-Hertha (Hrsg.), Bildung der Generationen (S. 387-399). Wiesbaden: Springer VS.

Löw, M. (2001). Raumsoziologie. Frankfurt am Main: Suhrkamp.

Mania, E. (2018). Weiterbildungsbeteiligung bildungsferner Gruppen in sozialraumorientierter Forschungsperspektive (1. Aufl.). Bielefeld: W. Bertelsmann.

Mania, E., Bernhard, C., \& Fleige, M. (2015). Raum in der Erwachsenen-/Weiterbildung. Rezeptionsstränge im wissenschaftlichen Diskurs. In C. Bernhard, K. Kraus, S. Schreiber-Barsch \& R. Stang (Hrsg.), Erwachsenenbildung und Raum. Theoretische Perspektiven - professionelles Handeln - Rahmungen des Lernens (S. 29-39). Bielefeld: W. Bertelsmann.

Martin, A., Schömann, K., Schrader, J., \& Kuper, H. (Hrsg.). (2015). Deutscher Weiterbildungsatlas. Bielefeld: W. Bertelsmann.

Martin, A., Schömann, K., \& Schrader, J. (2016). Der Einfluss der kommunalen Steuerung auf die Weiterbildungsbeteiligung - Ein Mehrebenen Modell mit Daten des Mikrozensus in Deutschland. Zeitschrift für Erziehungswissenschaft, 19(1), 55-82. https://doi.org/10.1007/s11618-015-0668-9.

Nugel, M. (2014). Erziehungswissenschaftliche Diskurse über Räume der Pädagogik. Wiesbaden: Springer VS.

Nuissl, E., \& Nuissl, H. (Hrsg.). (2015). Bildung im Raum. Baltmannsweiler: Schneider Verlag Hohengehren.

Robak, S., \& Rippien, H. (2015). Heidemann. In C. Pohlmann (Hrsg.), Bildungsurlaub - Planung, Programm und Partizipation. Eine Studie in Perspektivverschränkung. Frankfurt am Main: Peter Lang.

Schäffter, O. (1993). Die Temporalität von Erwachsenenbildung. Überlegungen zu einer zeittheoretischen Rekonstruktion des Weiterbildungssystems. Zeitschrift für Pädagogik, 39(3), 443-462.

Schemmann, M. (2006). Die Nähe zum Bildungsort zählt. Weiterbildungsbeteiligung zwischen sozialer Lage und Raum. Weiterbildung. Zeitschrift für Grundlagen, Praxis und Trends, (5), 28-31.

Schiersmann, C. (2006). Profile lebenslangen Lernens. Weiterbildungserfahrungen und Lernbereitschaft der Erwerbsbevölkerung. Bielefeld: W. Bertelsmann.

Schlüter, A. (2005). „In der Zeit sein...“. In A. Schlüter (Hrsg.), „, In der Zeit sein- “. Beiträge zur Biographieforschung in der Erwachsenenbildung (S. 9-18). Bielefeld: W. Bertelsmann.

Schmidt-Lauff, S. (2007). Zeit in der Erwachsenenbildung - Erwachsenenbildung in der Zeit. In G. Wiesner, C. Zeuner \& H. J. Forneck (Hrsg.), Empirische Forschung und Theoriebildung in der Erwachse- 
nenbildung. Dokumentation der Jahrestagung der Sektion Erwachsenenbildung der Deutschen Gesellschaft für Erziehungswissenschaft vom 21. bis 23. September 2006 an der Justus-Liebig-Universität in Gießen (S. 222-235). Baltmannsweiler: Schneider Hohengehren.

Schmidt-Lauff, S. (2008). Zeit für Bildung im Erwachsenenalter. Interdisziplinäre und empirische Zugänge. Münster: Waxmann.

Schmidt-Lauff, S. (Hrsg.). (2012). Zeit und Bildung. Annäherungen an eine zeittheoretische Grundlegung. Münster: Waxmann.

Schmidt-Lauff, S. (2018). Zeittheoretische Implikationen in der Erwachsenenbildung. In R. Tippelt \& A. von Hippel (Hrsg.), Handbuch Erwachsenenbildung/Weiterbildung (S. 319-338). Wiesbaden: Springer.

Schmidt-Lauff, S., \& Schreiber-Barsch, S. (2018). RaumZeit - Zeit und Raum in der Erwachsenenbildung. Call for Papers der Zeitschift für Weiterbildungsforschung. https://www.die-bonn.de/docs/ CfP_RaumZeit_1_2019_final.pdf

Schulenberg, W., Loeber, H.-D., Loeber-Pautsch, U., Pühler, S., Driesen, H., \& Scharf, W. (1978). Soziale Faktoren der Bildungsbereitschaft Erwachsener. Eine empirische Untersuchung. Stuttgart: KlettCotta.

Sixt, M., Bayer, M., \& Müller, D. (Hrsg.). (2018). Bildungsentscheidungen und lokales Angebot. Die Bedeutung der Infrastruktur für Bildungsentscheidungen im Lebensverlauf (1. Aufl.). Münster: Waxmann.

Stang, R. (2015). Lernräume in Bibliotheken. Optionen für eine offene Lerninfrastruktur. In C. Bernhard, K. Kraus, S. Schreiber-Barsch \& R. Stang (Hrsg.), Erwachsenenbildung und Raum. Theoretische Perspektiven - professionelles Handeln - Rahmungen des Lernens (S. 167-178). Bielefeld: W. Bertelsmann.

Stang, R., Bernhard, C., Kraus, K., \& Schreiber-Barsch, S. (2018). Lernräume in der Erwachsenenbildung. In R. Tippelt \& A. von Hippel (Hrsg.), Handbuch Erwachsenenbildung/Weiterbildung (S. 643-658). Wiesbaden: Springer.

Strauss, A., \& Corbin, J. (1996). Grounded Theory: Grundlagen qualitativer Sozialforschung. Weinheim: Beltz.

Tippelt, R., \& Reich-Claassen, J. (2010). Lernorte - Organisationale und lebensweltbezogene Perspektiven. REPORT - Zeitschrift für Weiterbildungsforschung, 33(2), 11-21. https://doi.org/10.3278/ REP1002W011.

Tippelt, R., Weiland, M., Panyr, S., \& Barz, H. (2003). Weiterbildung, Lebensstil und soziale Lage in einer Metropole. Studie zu Weiterbildungsverhalten und-interessen der Münchner Bevölkerung. Bielefeld: W. Bertelsmann.

Weidenhaus, G. (2013). Relationale Raumkonzeption. Hessische Blätter für Volksbildung. Zeitschrift für Erwachsenenbildung in Deutschland, 63(3), 212-219.

Wittpoth, J. (2006). Große Fragen, kleine Antworten. Probleme und Perspektiven der Weiterbildungsforschung am Beispiel der Beteiligungsregulation. In K. Meisel \& C. Schiersmann (Hrsg.), Zukunftsfeld Weiterbildung. Standortbestimmungen für Forschung Praxis und Politik (S. 53-68). Bielefeld: W. Bertelsmann.

Wittpoth, J. (2011). Beteiligungsregulation in der Weiterbildung. In R. Tippelt \& A. von Hippel (Hrsg.), Handbuch Erwachsenenbildung/Weiterbildung (5. Aufl. S. 771-788). Wiesbaden: Springer VS.

Witzel, A. (1982). Verfahren der qualitativen Sozialforschung: Überblick und Alternativen. Frankfurt am Main/New York: Campus.

Witzel, A. (2000). Das problemzentrierte Interview. Forum qualitative Sozialforschung, 1(1) https://doi. org/10.1007/978-3-8349-9441-7_29.

Wolff, J. (2005). Zeit für Erwachsenenbildung. Evangelische Erwachsenenbildung zwischen Zeit-Diagnosen und Frei-Zeit-Bedürfnissen. Göttingen: V \& R Unipress.

Zeuner, C., \& Faulstich, P. (2009). Erwachsenenbildung - Resultate der Forschung. Entwicklung, Situation und Perspektiven. Weinheim, Basel: Beltz.

Publisher's Note Springer Nature remains neutral with regard to jurisdictional claims in published maps and institutional affiliations. 